\title{
Impact of the Breakdown Behavior on Chinese Traditional Stewed Pork with Brown Sauce: Physical Properties Using Microstructural Analysis
}

\author{
Dengyong Liu, ${ }^{1}$ Yajun Deng, ${ }^{1}$ Ana Gong, ${ }^{1}$ Yaohui Han, ${ }^{1}$ and Hui-Min David Wang ${ }^{2,3,4}$ \\ ${ }^{1}$ College of Food Science and Technology, Bohai University, Food Safety Key Lab of Liaoning Province, \\ National \& Local Joint Engineering Research Centre of Storage, Processing and Safety Control Technology for Fresh Agricultural \\ and Aquatic Products, Jinzhou 121013, China \\ ${ }^{2}$ Graduate Institute of Biomedical Engineering, National Chung Hsing University, Taichung 402, Taiwan \\ ${ }^{3}$ Department of Medical Research, China Medical University Hospital, China Medical University, Taichung 404, Taiwan \\ ${ }^{4}$ College of Oceanology and Food Science, Quanzhou Normal University, Quanzhou 362000, China
}

Correspondence should be addressed to Dengyong Liu; jz_dyliu@126.com and Hui-Min David Wang; davidw@dragon.nchu.edu.tw

Received 30 December 2016; Revised 17 February 2017; Accepted 20 February 2017; Published 30 March 2017

Academic Editor: Jing Dai

Copyright (C) 2017 Dengyong Liu et al. This is an open access article distributed under the Creative Commons Attribution License, which permits unrestricted use, distribution, and reproduction in any medium, provided the original work is properly cited.

\begin{abstract}
The potential physics differences of Chinese traditional stewed pork during mastication were investigated. Ten subjects chewed and expectorated the fat and lean layers of stewed pork with brown sauce at different stages of mastication. The produced boluses were analyzed for their physical properties. The results suggested the subjects' saliva secretion and moisture content of the boluses during mastication increased significantly depending on subjects and food types studied $(P<0.05)$ and led to increase of bolus apparent particle size because of saliva uptake. Bolus first peak force tended to decrease significantly, whereas bolus flowability increased significantly during mastication $(P<0.05)$. Further, microstructure of boluses revealed series processing was conducted by comminution, aggregation, hydration, and dilution. The boluses ready-to-swallow possessed a higher flowability and a homogenetic matrix. Therefore, the changes in physics and microstructure of bolus contributed to dynamic texture perception of traditional Chinese stewed pork with brown sauce.
\end{abstract}

\section{Introduction}

During chewing, food breaks down, exposing food particles to saliva in the mouth. A swallowing will be triggered when the food transforms into a cohesive bolus [1]. Saliva is smooth and lubricates food particles, leading to an increase in bolus moisture $[2,3]$. The physical properties of the bolus can be changed by chewing sequences where the internal structure undergoes a dramatic transformation into a paste-like bolus with the amount of granules dispersed in the liquid solution of the saliva [4]. Texture perception at different stages of mastication can be changed by the addition of saliva in the formation of a bolus and affects its properties [5]. Oral physiology parameters and the perceived texture of all foods studied were likely to be influenced by adding water to food such as biscuits and rice cakes samples [3]. Therefore, determining individual saliva secretion at different stages during mastication is important to fully understand people texture perception.

The food breakdown path demonstrates the progressive reorganization of food structure during mastication, which is linked to individual texture perception, including the degree of structure, degree of lubrication, and chewing time $[6,7]$. Texture perception is the sensory manifestation of the food structure, and this reacts to the force applied to the bolus at different stages of mastication [8]. Therefore, the bolus particle size and mechanical properties related to the degree of structure could significantly influence texture perception in the mouth. Furthermore, bolus microstructure is another factor in evaluating the degree of structure during mastication [9]. 
Stewed pork with brown sauce is one the most special of Chinese traditional food. There were substantial intramuscular or subcutaneous fat in it and they maintained "primitive state." Once into the mouth, it will give a unique texture perception under mastication and saliva lubrication [10].

The objective of this study was to investigate the breakdown path of traditional Chinese stewed pork with brown sauce by measuring bolus moisture content (degree of lubrication), particle size distribution, and mechanical properties (degree of structure) at different stages of mastication. Bolus microstructure images during mastication were needed to characterize the internal structural changes by field emission scanning electron microscopy (FE-SEM).

\section{Materials and Methods}

\subsection{Stewed Pork with Brown Sauce Preparation}

2.1.1. Materials. Pork belly with a ratio of fat to lean of about four to six was used as the main material of stewed pork with brown sauce. Chives, ginger, star anise, and cinnamon were used as excipients. Soy sauce, fermented bean curd, and sugar were used as flavorings. Soybean oil and spirit were also used. The above materials were purchased from a local supermarket and used without further purification.

2.1.2. Stewed Pork with Brown Sauce Cooking Process and Preparation. The stewing process of fresh pork belly pieces: one kilogram of pork belly is cut into pieces $(35 \mathrm{~mm} \times 35 \mathrm{~mm}$ $\times 40 \mathrm{~mm}$ ) after washing and draining. The fresh pork belly pieces were oil fried 2.5 minutes at $150-160^{\circ} \mathrm{C}$. The obtained prepork belly pieces were stir fried for one minute with excipients in a wok, $50 \mathrm{~g}$ spirit was added and stir fried for one minute, flavorings ( $80 \mathrm{~g}$ Soy sauce, $40 \mathrm{~g}$ fermented bean curd, and $50 \mathrm{~g}$ sugar) were added and stir fried for two minutes, successively, and then one kilogram boiling water was poured and the stew was simmered for two hours.

We were aware that both the water content and fat content of the fat layer of stewed pork with brown sauce significantly differ from that of the lean layer. It is also true the physical properties of different locations on the stewed pork with brown sauce vary wildly. Of course, the mastication behavior and salivary secretion of different subjects vary as a result of the difference in physical properties of stewed pork with brown sauce. Therefore, for the sake of scientific accuracy and rigorousness, the fat layer and the lean layer of stewed pork with brown sauce were analyzed separately throughout this investigation.

After the end of cooking, blotting paper was used to remove the juices and other debris on the surface of the meat, the pigskin was cut off, and the fat layer and the lean layer were cut into squares of $20 \mathrm{~mm} \times 20 \mathrm{~mm} \times 5 \mathrm{~mm}$, respectively (weigh $3.3 \pm 0.2 \mathrm{~g}$ ). Stewed pork with brown sauce was cooked and diced the day before the experiments. The blocks were stored under seal at $4^{\circ} \mathrm{C}$ and used within one day of preparation. These products were drain heated for 3-5 minutes just before sensory testing.

\subsection{Subjects}

2.2.1. Subject Selection. Ten healthy subjects were picked from among 36 school students majoring in Food Science and Engineering for the study, according to their motivation. The subjects (five women and five men) ranged from 18 to 25 years of age. All of the subjects had good dental health. None of them were smoking nor disliked the food, which might have affected the saliva secretion or taste perception. Subjects received no financial award for their time but a small packet of snacks was given as a gesture of thanks at the end of each session.

2.2.2. Training Sessions. Five experiments were carried out; the same ten subjects participated in all of the experiments. None of the subjects had any previous sensory analysis experience and were accepted as untrained subjects. Each subject attended a preliminary session to become familiar with elementary knowledge of sensory analysis and procedures. The first four training sessions focused on the identification of subject mastication behavior while eating. To prevent hunger or the impact of food digestion on chewing and swallowing abilities, subjects were asked not to eat 90 minutes before the sensory experiment. Each subject was required to chew and swallow each sample (both the fat and lean layer of stewed pork with brown sauce) in a natural way until the swallowing point was reached. The mastication time of bolus ready to be swallowed was recorded using a stopwatch to characterize the mastication behavior of the subjects and each product. The number of cycles was recorded for each subject while chewing two different samples several times.

2.3. Food Bolus Collection. In this experiment, both only fat parts and only lean parts of stewed pork with brown sauce were provided for the subjects. The same group of volunteers was asked to chew each sample, including both fat and lean layers of stewed pork for the bolus examinations. After swallowing, the time of swallowing was recorded for each subject and each food type. To clarify the breakdown behavior of food bolus in the entire masticatory process, boluses were collected after mastication at 20\%, 40\%, 60\%, 80\%, and $100 \%$ of the total chewing time for physical analysis.

Physical and chemical analysis experiment required subjects to chew but not swallow the food samples. Each participant subject spit out the main bolus at different stages of chewing materials, and we did not collect the oral residue materials The same individual group was asked not to clean their oral cavities to prevent water and meat residues from being in the mouth, which may lead to an increase of saliva secretion to change the structure of the bolus. After all of the food boluses were collected, the subjects rinsed their mouth with water. Less than 2 minutes was given for chewing each sample. Each subject chewed the fat layer and the lean layer of stewed pork with brown sauce and spit out the food bolus at least three times for each sample. The masticatory experiment could not exceed 60 minutes. Boluses were analyzed immediately following collection to prevent water evaporation and the role of enzymes in saliva. 


\subsection{Food Bolus Evaluation}

2.4.1. Moisture Content and Saliva Content Analysis. The moisture contents of food samples and boluses on a wet weight basis were determined by measuring the weight difference before and after drying at $105^{\circ} \mathrm{C}$ in an air oven (AACC method 45-30). Weighing and recording took place several more times at hourly intervals to achieve a constant weight, which is defined as a weight difference of no more than $2 \mathrm{mg}$. The moisture content on a dry basis, which is the ratio between the quality of water including saliva and the dry materials of bolus, was estimated using the formula $\mathrm{MC}_{\mathrm{db}}=\mathrm{MC}_{\mathrm{wet}} /(1-$ $\left.\mathrm{MC}_{\text {wet }}\right)$.

Saliva added (SA) per gram of dry food was calculated using the formula $\mathrm{SA}=\mathrm{MC}_{\mathrm{db} \text {,bolus }} / \mathrm{MC}_{\mathrm{db} \text {,food }}$, where $\mathrm{MC}_{\mathrm{db}}$ bolus represents the moisture content of the collected bolus on a dry basis, and $\mathrm{MC}_{\mathrm{db}}$ food represents the dry basis food moisture content before chewing. The bolus collected from the mouth was not complete, excluding oral residue. However, we believe the recovered mass could be representative for the general analysis. The recovery calculation ratio was to measure the removing dry mass of food from the bolus. In this experiment, the average recovery ranged between $63 \%$ and $87 \%$ for the fat layer of stewed pork with brown sauce and about $89 \%$ to $116 \%$ for that of the lean layer.

2.4.2. Particle Size Distribution of Bolus. Following expectoration, the bolus was spread out on a centrifuge tube and the particle parameters were analyzed. We tried to dilute a small amount of the recently spit out bolus using $80 \mathrm{~mL} 0.1 \mathrm{~g} / \mathrm{mL}$ Sodium dodecyl sulfate (SDS) solution to prevent agglomeration of the lipomicron particles in the bolus emulsion resulting from steps taken during sample preparation. The suspension was slightly agitated with a glass rod before measuring to maintain particles system in a dispersed stabilized state, relatively. Bolus emulsion as an aqueous suspension diluted using deionized water with SDS was loaded into the Laser particle size analyzer (BT-9300ST, Bettersize Instruments Ltd, China) for measuring particle size and particle size distribution. Mie theory as an optical mode with the device for supporting software was selected for these measurements. Deionized water was used as a dispersant for particle size analysis. The stewed pork with brown sauce samples and water had refractive indexes of 1.52 and 1.33, respectively. This is expressed by the refractive index of the instrument between 5 and 15 as the boundary value. The particle size distribution is $0.1-1000 \mu \mathrm{m}$. Representative aliquots of the samples (the fat layer, $n 3$, and the lean layer, $n$ 3) obtained after mastication at each mastication stage were poured into the automated sample dispersion unit filled with SDS solution. For each sample, ten consecutive measurements of $60 \mathrm{~s}$ duration were conducted. The apparent particle size was quantified in terms of $\mathrm{d} 50$, representing the particle size of volume accumulated value up to 50 percent of the total volume of all particles present in the distribution.

2.4.3. Characterization of Mechanical Bolus Properties Using Two Cycle Penetration Tests (TCPT). A two-cycle penetration test (TCPT) on the bolus, which was adapted from the modified Texture Profile Analysis described by Peyron et al. [11], was used to measure the mechanical properties of the bolus spit out immediately. Each bolus was collected into a cylindrical glass vial for each subject and the lean layer of stewed pork with brown sauce sample. We gently squeezed the bolus using the back of a small spoon to achieve relatively uniform surface smoothing. A cylindrical mass approximately $5 \mathrm{~mm}$ in height and $25 \mathrm{~mm}$ in diameter was obtained by emptying the contents of the glass vial and loading it into a Texture Analyzer (TA.XT plus, Stable Micro Systems-SMS) with a $5 \mathrm{~g}$ load cell for TCPT consisting of two successive uniaxial compression tests. The fat layer samples were obtained in triplicate by a similar procedure for its serious loss mass during mastication. A cylindrical steel probe of $50 \mathrm{~mm}$ in diameter was used to perform a double penetration test over $75 \%$ of their height at a constant speed of $1 \mathrm{~mm} / \mathrm{s}$. Retrieval of the probe was at a speed of $2.0 \mathrm{~mm} / \mathrm{s}$ and the interval time between two compression tests was five seconds. Data acquisition was carried out at a sample frequency of 200.00 ps. From the TCPT force-time curves, resilience, adhesiveness, and flowability parameters were obtained as the first peak force [11-13]. First peak force represents bolus firmness. Resilience represents the degree of bolus springing back to its initial state and shape following one penetration cycle. Adhesiveness represents the adhesion of the bolus after one penetration cycle. Flowability represents the degree to which the bolus returns to its initial state and shape after one compression cycle from a combination of flowing and springing.

2.4.4. Microstructural Analysis. The microstructure of the ready-to-swallow bolus was studied with S-4800 field emission scanning electron microscopy (FE-SEM, Hitachi Ltd). Following collection, food boluses were put into a refrigerator at $4^{\circ} \mathrm{C}$ overnight to achieve a relatively stable form. To minimize the tissue damage of each bolus, sharp knife blades were used to cut the samples to achieve a well-defined $2 \mathrm{~mm}^{3}$ block for microstructural analyses. All of the sections were allowed 24 hours to immobilize using 2.5\% glutaraldehyde at $4^{\circ} \mathrm{C}$, which was prepared with $0.1 \mathrm{M}$ phosphate buffer solution (PBS, pH 6.8). The bolus blocks were washed three times using PBS for ten minutes each time. After washing, gradient elution was applied for ten minutes to elute the blocks, using 50\%, 70\%, 80\%, and 90\% ethanol, respectively. Bolus block dehydration was needed three times using absolute ethyl alcohol, lasting ten minutes each time. Then trichloromethane was used to wash bolus blocks again after one hour. Further dehydration was needed using isometric alcosol covering trichloromethane and tertiary butyl alcohol and tertiary butyl alcohol alone, with each treatment continuing for 15 minutes in succession. Finally, vacuum freeze drying was used to sublime the moisture of the bolus blocks using a vacuum freeze drier (Free Zone 2.5, Labconco, USA). Before recording the microstructural images of bolus blocks, the fractured surface was sputter-coated with gold (E-1045 Vacuum sputtering apparatus, Hitachi Ltd). The prepared sample was transferred to the cold stage of the SEM chamber and examined with an amplification of $10 \mathrm{k}$ at low beam voltage $(5 \mathrm{kV})$. 
2.5. Statistical Analysis. Each sample was measured at least three times by repeated and statistical analysis performed using SPSS 20.0 software. All results are presented as means with their standard errors, unless otherwise stated. ANOVA analysis was used to test variance analysis. Data which conformed to the normal distribution method of multiple comparisons used Duncan and those that did not conform used the Kruskal-Wallis test. Pearson product-moment correlations were used to determine the relationships at $P<0.05$ and $P<0.01$ significance levels. Graphic processing was needed using Origin 8.6 software.

\section{Results and Discussion}

\subsection{Mastication Behavior Management}

3.1.1. Mastication Duration. The time required for mastication of a piece $(20 \mathrm{~mm} \times 20 \mathrm{~mm} \times 5 \mathrm{~mm})$ of stewed pork with brown sauce ( $n=$ ten subjects, triplicate) was determined for normal eating. Owing to individual differences, the duration from the first bite until triggering a swallowing significantly differed for each subject and each food style. The normal eating time of the fat layers of stewed pork with brown sauce averaged $12.24 \pm 2.72 \mathrm{~s}(6.02-13.07 \mathrm{~s})$ compared to $27.76 \pm$ $6.59 \mathrm{~s}(15.20-27.80 \mathrm{~s})$ for the lean layer pieces. Mastication time significantly differed for subjects $(P<0.05)$ and was significantly lower for the fat layers $(P<0.001)$ compared to pieces of the lean layer. The reason behind the difference in chewing time between the fat and lean parts could be the fat content in meat. Fat could lubricate the mouth and cut the chewing time. Therefore, different chewing times were chosen for $100 \%$ mastication duration for each subject and each food style.

3.1.2. Saliva Added Analysis. The saliva added differences in boluses between the stewed pork with brown sauce food samples at different mastication stages are determined (Figure 1). Different English letters (A and B; $\mathrm{A}^{\prime}$ and $\mathrm{B}^{\prime}$ ) indicated that there was significant difference between each groups in different process $(P<0.05)$. The sample weight was $3.3 \pm 0.2 \mathrm{~g}$, and the tallish in bar-pattern was to present the big standard deviation because of the individual variation in chewing behavior. Saliva tended to increase from the beginning of mastication to swallowing for both food samples. Saliva for each subject was significantly higher for the lean layers in the latter mastication stage (80\% mastication) compared to the early mastication stage of $20 \%$ mastication but did not significantly differ with the swallowing point (Figure 1). The saliva added of subjects for the fat layers was relatively complex. The possible reason could be that fat may suppress saliva secretion [14]. A tendency of increasing saliva added was already present for the fat layers during the earlier mastication stage before $40 \%$ mastication, when the food structure was relatively complete and broken into pieces with different particles. The saliva was little changed when the subject continued chewing to $80 \%$ mastication and the food sample underwent further fragmentation and aggregated into a homogeneous bolus. During the latter part of the chewing sequence to reach a swallowing point, the saliva significantly increased again and

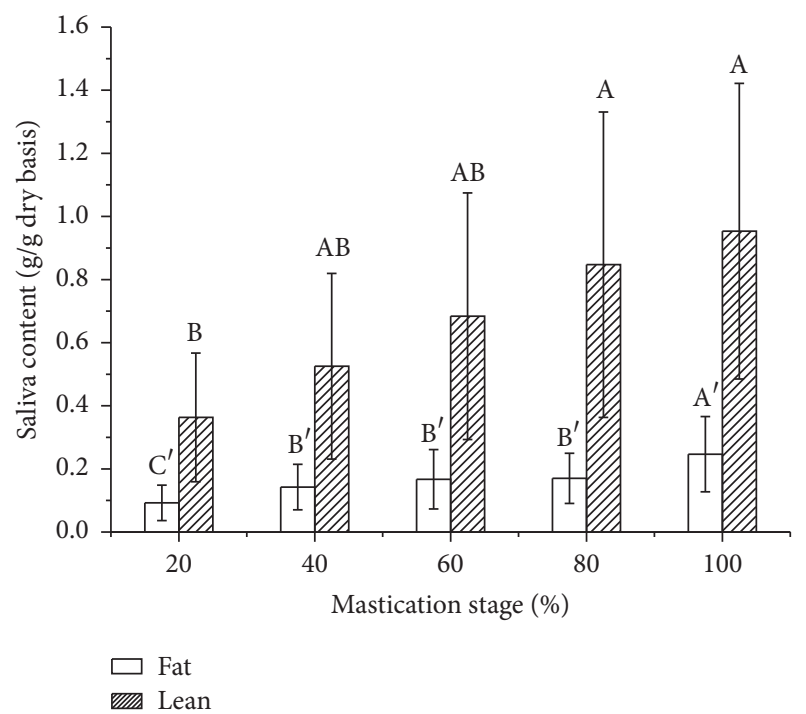

FIgURE 1: The saliva was secreted by participants during the mastication. Different letters indicate that there was significant difference in different process $(P<0.05)$.

a swallowing could be triggered with higher moisture content. Different changes in each subject of the saliva during different mastication stages for salivary secretion may be affected by the dispersion of food ingredients or taste substances [6]. In addition, food texture could be another factor significantly influencing subject saliva secretion [15]. Another interesting finding was the subject significantly affected bolus moisture content (62-85\%) and saliva incorporated [16]. Perceived food texture could also be affected by the mastication process. The saliva of the subjects could directly influence both bolus moisture and perceived texture [2].

\subsection{Bolus Properties}

3.2.1. Bolus Moisture Content. The addition of saliva to food samples with chewing could cause an increase of bolus moisture content and cluster a large number of particles into a cohesive bolus. Figure 2 shows the moisture content of the two different food boluses in a dry basis increased significantly during mastication $(P<0.05)$. These were explained by saliva secretion with chewing for each subject. The rate of bolus moisture content growth in the early mastication was faster than the latter duration, which agreed with the condition of the rapid saliva absorbed with the low initial moisture content of unchewed food samples during the early stage of mastication. The stages during the early and latter mastication duration were the main stages of bolus moisture content changed when subject saliva secretion clearly varied. Similar moisture contents for the lean layer boluses were measured for boluses at $80 \%$ and $100 \%$ mastication, implying saliva absorption stabilized towards the end of mastication. The moisture content of the fat layer samples at the swallowing point was significantly higher than the prior mastication stages. This was most likely a result of the dilution of bolus with a relatively large amount saliva secretion. A difference 


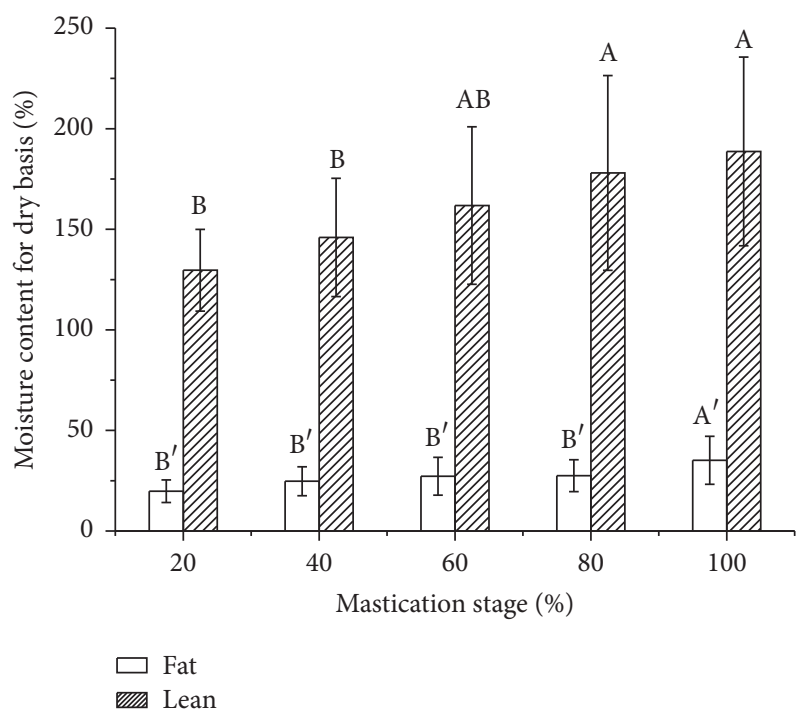

Figure 2: The moisture content of bolus during the mastication.

between the two boluses could be observed at the same mastication stage and the fat layer bolus tended to be lower than the lean layer samples at each chewing sequence. The point of swallow could be achieved once the food bolus had absorbed a certain mass of water [2].

3.2.2. Bolus Particle Size Distribution. Apparent particle size represents two different styles of stewed pork with brown sauce particles and the saliva-food agglomerates, which varied in size during mastication. Figure 3(a) compared the rate of change in the bolus structure of the two different meat boluses at different mastication stages in terms of apparent particle size. As a single cluster was formed by the agglomeration of several small particles, the diameter of the fat layer fragments in the bolus increased insignificantly over the course of the eating time. Apparent particle size in the latter mastication was higher than at the early stages for the lean layer bolus. This could be explained by the saliva rate being added to the food bolus structure [17]. Compared with the masticated lean layer bolus, the fat layer boluses are composed of particles of smaller size. Thus, the mean particle sizes are $231 \mu \mathrm{m}$ (SEM 43) and $110 \mu \mathrm{m}$ (SEM 34) for the lean and fat samples at the swallowing point, respectively. These may be related to the structure and initial food moisture content [9].

Particle size distribution of the two different food bolus samples was multimodal and broad (Figures 3(b) and 3(c)). The fat layer samples displayed clear double peaks at particle sizes of $2.154 \mu \mathrm{m}$ and $183.8 \mu \mathrm{m}$ compared to the lean layer samples. The main peaks of the distribution profile were not changed in terms of the height and position. This indicated subject mastication could not arouse significant particle disintegration for both samples. Small changes could be observed across the entire range of particle size distribution, possibly owing to internal structural changes or the local saliva content in the bolus [9].

The apparent size of the bolus particles for the fat layer increased with more chewing cycles, especially at the swallowing point (Figure 3(a)). There may be a third peak at the particle size distribution of $476.6 \mu \mathrm{m}$ for the fat layer samples at the swallowing point (Figure 3(b)). The lean layer samples may display a third peak at $428.7 \mu \mathrm{m}$ at the early mastication stage (Figure 3(c)). This could be explained by the food structure possibly affecting the chewing behavior of each subject [18]. For the rich muscle fibers solids, the lean layer samples more easily produced several large crumbs compared to the fat layer samples. As with many other semisolid foods $[12,13,19]$, the fat layer samples produced a large number of small-sized particles measured in the early mastication stages, accounting for most of the bolus, thereby suggesting comminution dominated the initial stages of chewing. As mastication continued, a greater fraction of smaller and rounder fragments agglomerated into large particles with saliva access into the food bolus.

3.2.3. Mechanical Properties of Bolus. The mechanical properties of the expectorated bolus were determined by TCPT, including first peak force, flowability, resilience, and adhesiveness (Figure 4). First peak force represents bolus firmness. The first peak force of the two different food boluses decreased with chewing cycles and was significantly higher for the lean layer food samples $(P<0.05$, Figure $4(a))$. As mentioned earlier in Section 3.2.2, the apparent particle size of the food bolus increased with chewing time. These variation tendencies for the two different mechanical properties of the bolus could be inter-explained. Bolus with a lower rigidity was likely to show a larger apparent particle size than harder foods [20]. The food boluses from the lean layers were found to form clusters faster (at 60\% mastication) that may increase the force needed to compress the bolus. The decreased bolus first peak force was not significant during 60\% towards $100 \%$ mastication. This showed first peak force for the two different boluses could reach a plateau at the swallowing point. This was most likely a result of the melted fat due to high oral temperature and mastication. Flowability was relevant to a combination of flowing and springing behavior during the TCPT. Increased flowability of the two different boluses was observed as mastication continued and it was significantly lower for the fat layer food samples $(P<0.05$, Figure $4(\mathrm{~b}))$. The two different food samples possessed a slightly higher value of flowability at $20 \%$ mastication than at $40 \%$ mastication. This may be due to the saliva on the surface of the food sample without fusion into the bolus. The flowability of the fat layer bolus seemed to increase towards a target value with the lean layer bolus beginning from the $20 \%$ mastication stage. Another interesting finding was that the flowability of the lean layer bolus during the mastication stages after $60 \%$ mastication was lower than before. This may be why bolus formation was done at $60 \%$ mastication. Different flowability values between two boluses tended to decline. As mastication finished, two different food samples had a flowability value between 0.47 and 0.52 . This demonstrated the flowability of the food bolus converged to a value at the end of oral processing to ensure a comfortable and safe swallow. This result was consistent with other research. The cohesiveness of the food bolus was assumed to be a determining factor to trigger swallowing, which was similar to flowability measured 


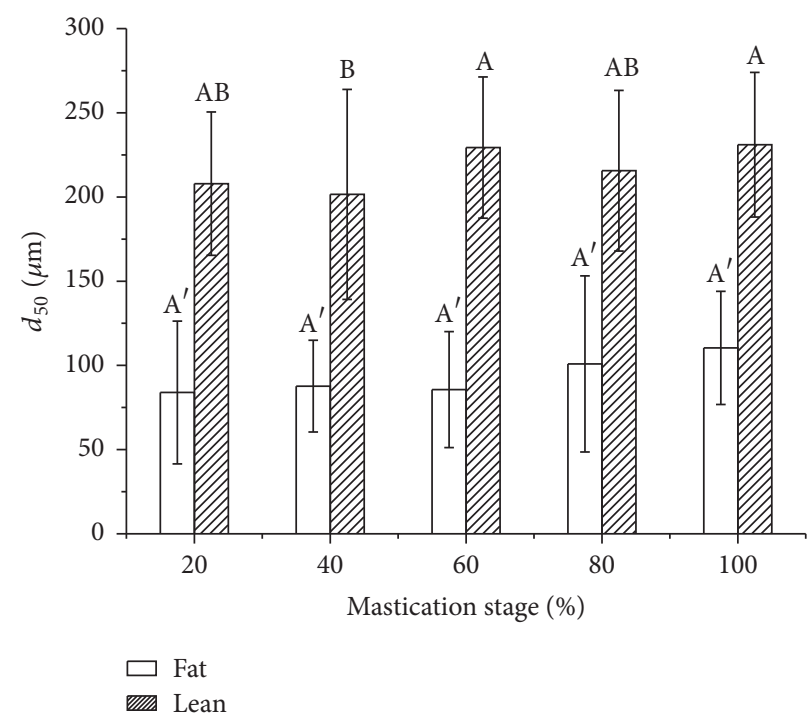

(a)

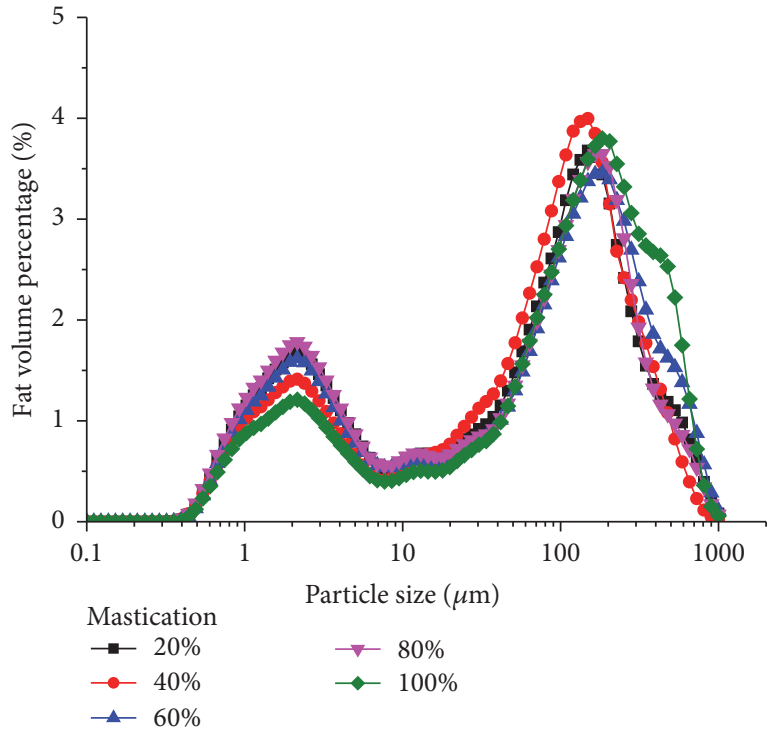

(b)

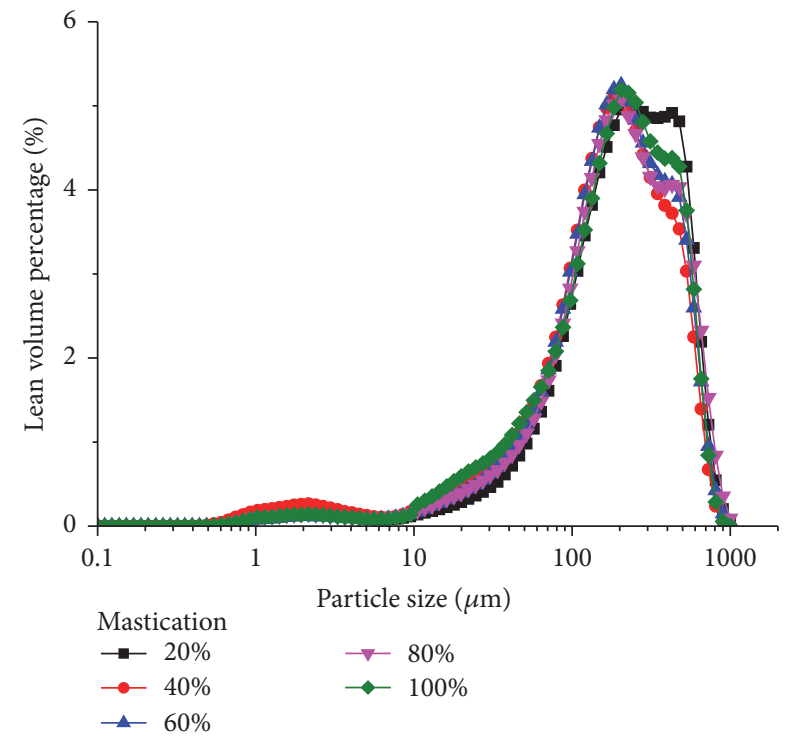

(c)

FIGURE 3: The apparent bolus particle size during the mastication (a); the particle size distribution of the fat layer (b); and the lean layer (c).

by modified TPA [21]. More precisely, a target value of flowability was needed to ascertain a swallowing point rather than a target value of cohesiveness [12, 13, 22]. Resilience did not vary significantly during mastication and was lower at $80 \%$ and $100 \%$ mastication compared to the early stages of chewing (Figure 4(c)). The fat layer food samples that broke down in the bolus were less resilient than the lean layer food samples. The fat layer samples were easier to break into pieces and would slightly resist under the first compression by the TCPT test compared to the lean layer boluses containing large fragments. The resilience of the bolus was closely related to elasticity, which was the dominant attribute perceived in the middle phase of mastication $[12,13]$. The adhesiveness of the food bolus increased with chewing cycles and was significantly higher during the later chewing sequences $(P<$ 0.05 , Figure $4(d))$. The adhesiveness of the fat layer food samples was a lower value than the fat layer boluses during mastication. The initial chewing values of adhesiveness for the fat layer samples were almost the same as the lean layer samples. After $20 \%$ period time of oral chewing, the adhesiveness of the lean layer samples increased larger than the lipid samples. These could be explained by more saliva secretion not blending with high concentrations of fat and creating a liquid phase surrounding the fat layer fragments, resulting in a softer and more flowable bolus rather than a mutually adhesive bolus. A potential correlation between the bolus mechanical property of adhesiveness and sensory perception of stickiness has been hypothesized.

3.2.4. Bolus Microstructural Analysis. The micrographs of two different food boluses showed the myofibrillar protein and pieces of fat were significantly affected by mastication 


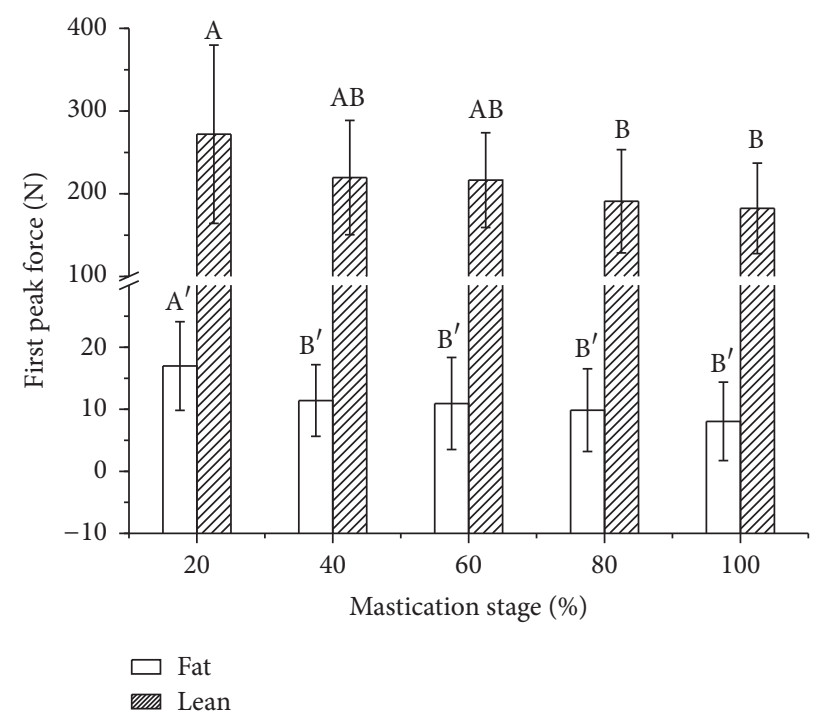

(a)

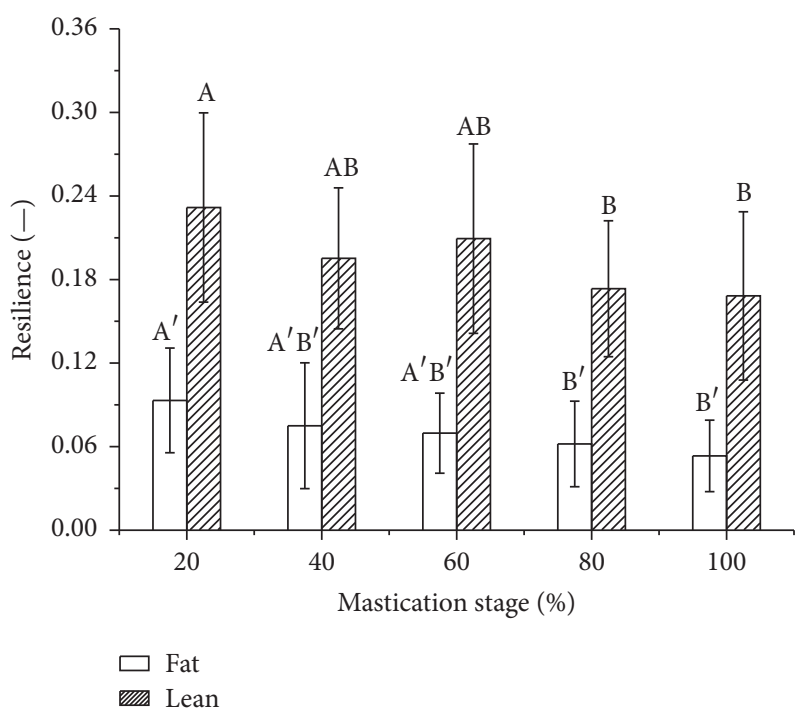

(c)

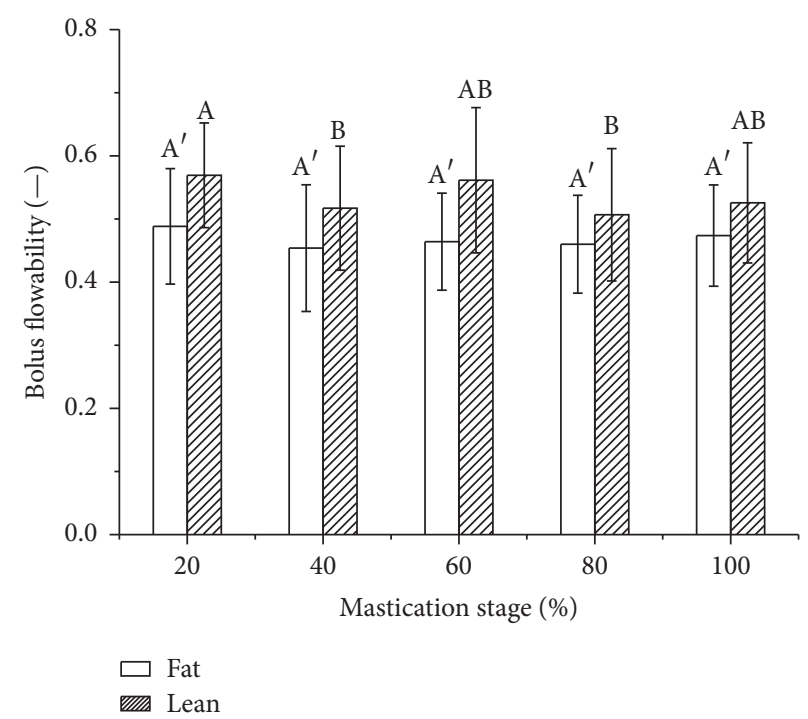

(b)

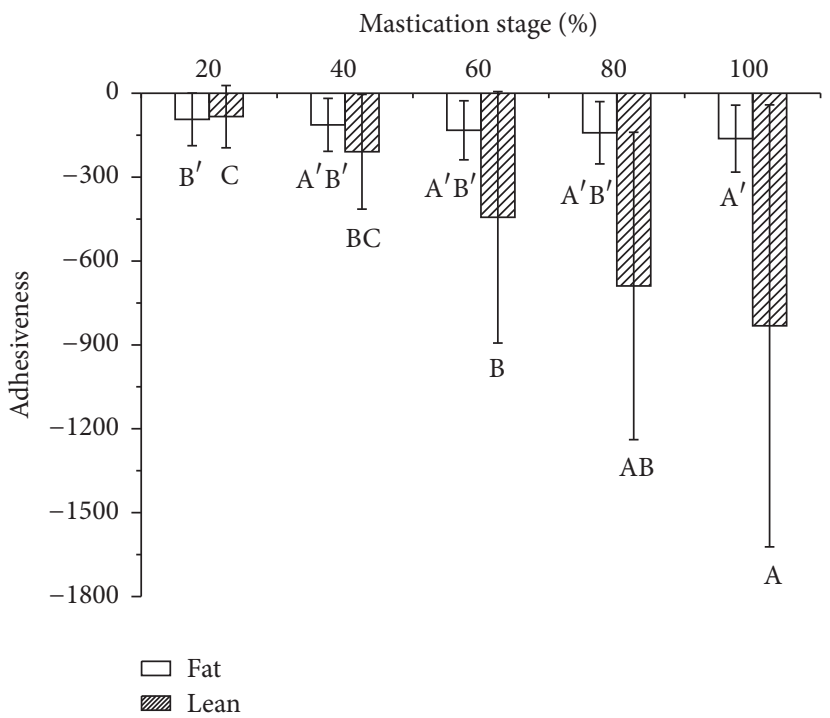

(d)

FIGURE 4: The first peak force of bolus mechanical properties during mastication (a); the flowability (b); the resilience (c); and the adhesiveness (d).

behavior. All FE-SEM images (Figure 5) of the two different boluses during mastication showed cross-sectioned fatprotein granules, but they differed in the structure of the matrix surrounding them. Some images implied a glassy, brittle phase was present between the granules, while others indicated a porous network surrounded them. These results were similar to the mastication conditions of biscuits bolus [17]. This could be explained by oral chewing behavior, including gelling and emulsification with mastication breakdown and saliva secretion. The original food samples were uniform and had low moisture content without chewing. The food samples then turned inhomogeneous with saliva blended, but it did not mix perfectly with proceeding mastication till the middle duration stage. The formation of a paste-like bolus which was relatively homogeneous and cohesive could be achieved after a long chewing time.

Figure 5 shows that the food samples underwent comminution and particle aggregation, hydrated and diluted with saliva. On a microstructural level, the images of the two different food boluses at $20 \%$ mastication were similar to the original food samples because of the small amount of saliva added and few chewing cycles conducted, while, in the next mastication stages, the degree of degradation for the two styles of bolus differed according to the different chewing times and different masticatory forces [23]. For the fat layer food samples, the small amount of saliva secreted varied locally in the bolus. Mastication transformed the solid food samples into a solid-liquid system and a lamellar mixing dominated 

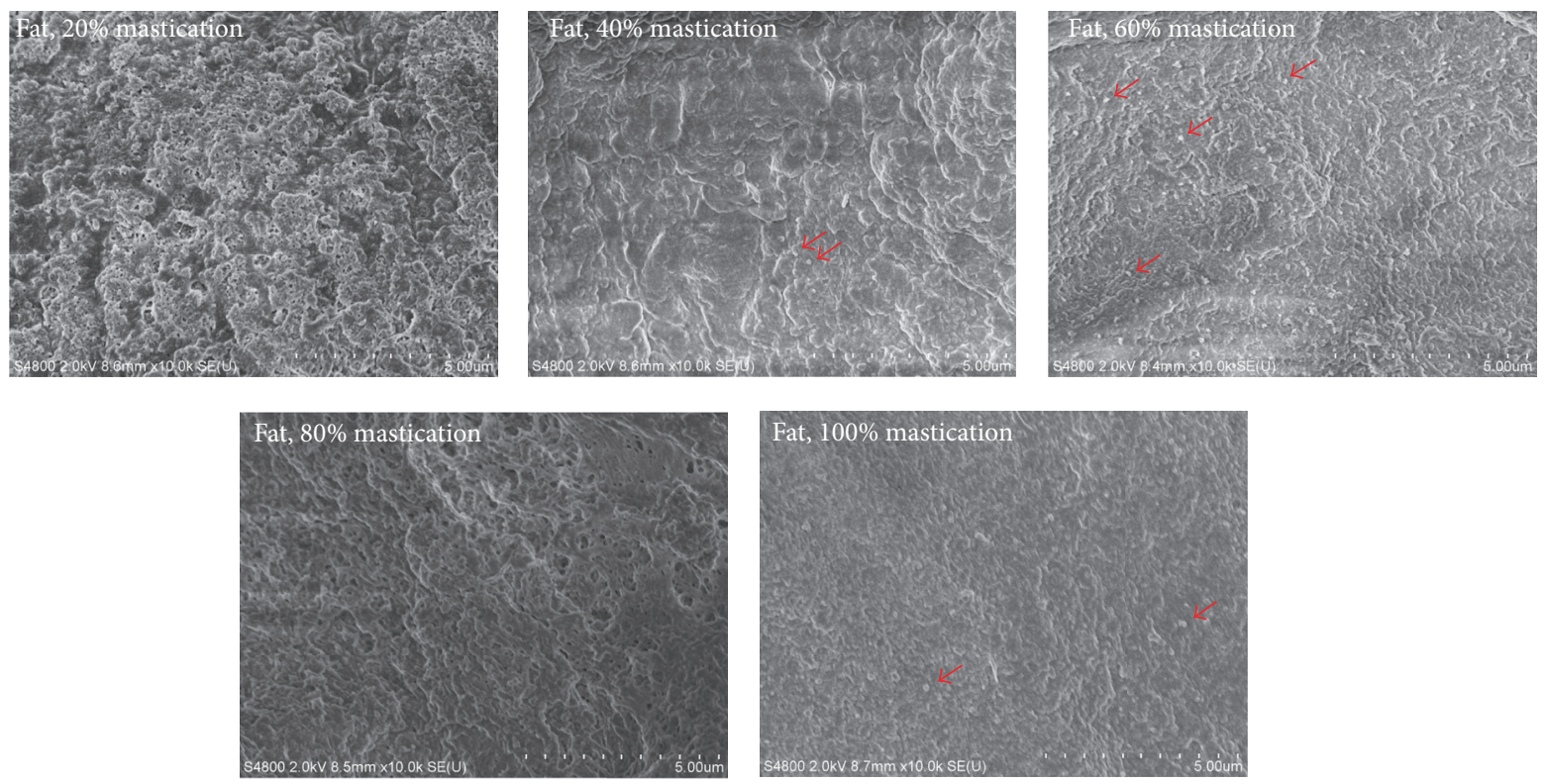

$\longleftarrow$ Fat granule
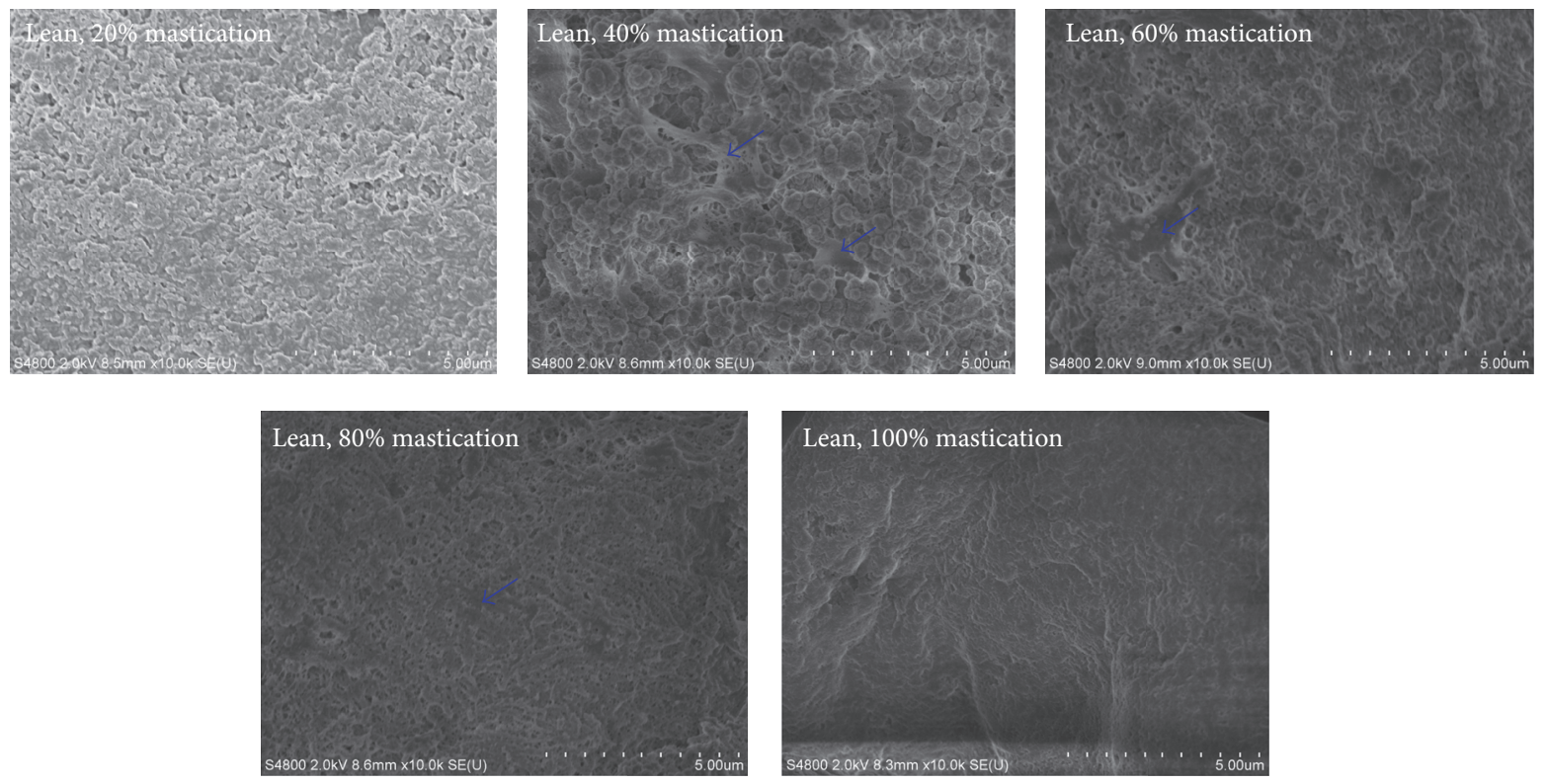

$\measuredangle$ Glassy phase

FIGURE 5: FE-SEM images of food boluses during the mastication. ${ }^{*} 20 \%=$ the mastication of $20 \%$ and $100 \%=$ the swallowing point.

during $40 \%$ mastication. When chewing to $60 \%$ mastication, many single fat particles which separated from one another solubilized in the saliva and dispersed into the solid matrix. This indicated that the food boluses were broken down to the level of single fat particles held in a continuous phase of saliva at subsequent chewing stages. This displayed the fat granules in the bolus with a typical bimodal size distribution more intuitively and was responsible for the results in Section 3.2.2. However, the impression of a porous network in the regions of the microstructural image was observed at $80 \%$ mastication. This was most likely a result of water loss which led to saliva secretion, neglecting the small loss of oil melted by oral temperature with chewing time. A continuous phase of solid matrix was reached at the swallowing point. There were differences in the lean layer food samples compared with the fat layer food samples for few particles lost and different time for bolus formation. Figure 5 shows that the saliva and bolus matrix were mixed and more homogeneous for the lean layer 
boluses during mastication time, especially from $40 \%$ mastication to the swallowing point. The proposed inhomogeneity of the bolus before the swallowing point provides a model for the type of mixing involved in mastication. Chaotic mixing according to the subject's individual chewing sequence could decrease heterogeneity by repeatedly stretching and folding the inhomogeneous layers [17]. The large-scale saliva gradually narrowed the long object and entered the food continuous phase. A homoplasmic condition of was also approached at the swallowing point. The microstructural images of the two different food boluses were entirely different for different internal structural changes and saliva distribution. It is known that individual texture perception is closely related to bolus structure [24]. The different parts of stewed pork with brown sauce could contribute to different textural perception.

\section{Conclusions}

The saliva secretion of the subject and the bolus physical properties including apparent particle size and bolus flowability and adhesiveness increased significantly during mastication for the two different parts of stewed pork with brown sauce. The physical properties of the bolus and microstructure images were found to be changed during mastication, which could be explained by increased bolus moisture content and chewing behavior (time and saliva secretion). In contrast, bolus first peak force significantly decreased with chewing cycles. The physical properties of the boluses for the two different types performed a similar trend compared with phases prior to mastication. Internal microscopic changes resulted in transformation of the macroscopic physical properties of food bolus. The microstructure of the two different boluses experienced the processes of comminution, aggregation, hydration, and dilution. However, microstructural changes of food boluses were more complex in the middle stages of mastication. The boluses ready to be swallowed possessed a higher flowability and a homogenetic matrix of microstructure, respectively. The microstructural images of the two different styles of food bolus just before swallowing suggested that the "paste phase" of the bolus is primarily composed of individual myofibrillar protein isolate particles or fat globules suspended in a continuous liquid solution of saliva.

From these results, we could hypothesize that the food bolus needs to incorporate enough saliva to be perceived as fluid enough to be swallowed. Saliva secretion mainly managed the water absorption of the food boluses and the individual mastication behavior, especially the chewing time. The saliva was incorporated into the food granules, determining the main physical properties of the food bolus. The physical properties of the fat layer boluses were significantly lower compared to the lean layer sample, including the apparent particle size and mechanical properties. Dynamic textural perception of the food bolus was found to be linked to the physical properties and dynamic evolution of bolus properties in the mouth [8]. Therefore, the two different parts of stewed pork with brown sauce contributed to individuals' dynamic textural perception.

\section{Additional Points}

Practical Applications. Chewing behavior is essential for the underlying physical properties involved in the transformation of the food to a cohesive bolus for swallowing and is found to be linked with the food texture perception during mastication. Hence, texture perception cannot be fully understood without analyzing bolus structure. Understanding changes of bolus physical properties, the role of food structure and effects on sensory perception can be applied in computational models of food breakdown and flavor release. This knowledge will allow "primitive state" fat-dependent meat food structures to be designed to enhance sensory appeal or follow a particular breakdown path in health food applications.

\section{Conflicts of Interest}

The authors declare that there are no conflicts of interest regarding the publication of this article.

\section{Acknowledgments}

This work was supported by National Natural Science Foundation Surface Project (31571861).

\section{References}

[1] J. Chen, "Food oral processing-a review," Food Hydrocolloids, vol. 23, no. 1, pp. 1-25, 2009.

[2] L. Motoi, M. P. Morgenstern, D. I. Hedderley, A. J. Wilson, and S. Balita, "Bolus moisture content of solid foods during mastication," Journal of Texture Studies, vol. 44, no. 6, pp. 468479, 2013.

[3] K. Shiozawa and K. Kohyama, "Effects of addition of water on masticatory behavior and the mechanical properties of the food bolus," Journal of Oral Biosciences, vol. 53, no. 2, pp. 148-157, 2011.

[4] C. Loret, M. Walter, N. Pineau, M. A. Peyron, C. Hartmann, and N. Martin, "Physical and related sensory properties of a swallowable bolus," Physiology and Behavior, vol. 104, no. 5, pp. 855-864, 2011.

[5] L. Engelen, P. A. M. van den Keybus, R. A. de Wijk et al., "The effect of saliva composition on texture perception of semisolids," Archives of Oral Biology, vol. 52, no. 6, pp. 518-525, 2007.

[6] P. J. Lillford, "The importance of food microstructure in fracture physics and texture perception," Journal of Texture Studies, vol. 42, no. 2, pp. 130-136, 2011.

[7] M. Panouillé, A. Saint-Eve, C. de Loubens, I. Déléris, and I. Souchon, "Understanding of the influence of composition, structure and texture on salty perception in model dairy products," Food Hydrocolloids, vol. 25, no. 4, pp. 716-723, 2011.

[8] A. Saint-Eve, M. Panouillé, C. Capitaine, I. Déléris, and I. Souchon, "Dynamic aspects of texture perception during cheese consumption and relationship with bolus properties," Food Hydrocolloids, vol. 46, pp. 144-152, 2015.

[9] G. Mandalari, M. M.-L. Grundy, T. Grassby et al., "The effects of processing and mastication on almond lipid bioaccessibility 
using novel methods of in vitro digestion modelling and microstructural analysis," British Journal of Nutrition, vol. 112, no. 9, pp. 1521-1529, 2014.

[10] T. van Vliet, G. A. van Aken, H. H. J. de Jongh, and R. J. Hamer, "Colloidal aspects of texture perception," Advances in Colloid and Interface Science, vol. 150, no. 1, pp. 27-40, 2009.

[11] M.-A. Peyron, I. Gierczynski, C. Hartmann et al., "Role of physical bolus properties as sensory inputs in the trigger of swallowing," PLoS ONE, vol. 6, no. 6, Article ID e21167, 2011.

[12] M. Devezeaux de Lavergne, J. A. M. Derks, E. C. Ketel, R. A. de Wijk, and M. Stieger, "Eating behaviour explains differences between individuals in dynamic texture perception of sausages," Food Quality and Preference, vol. 41, pp. 189-200, 2015.

[13] M. Devezeaux de Lavergne, F. van de Velde, M. A. J. S. van Boekel, and M. Stieger, "Dynamic texture perception and oral processing of semi-solid food gels: part 2: impact of breakdown behaviour on bolus properties and dynamic texture perception," Food Hydrocolloids, vol. 49, pp. 61-72, 2015.

[14] G. Feron, C. Ayed, E. M. Qannari, P. Courcoux, H. Laboure, and E. Guichard, "Understanding aroma release from model cheeses by a statistical multiblock approach on oral processing," PLOS ONE, vol. 9, no. 4, Article ID e93113, 2014.

[15] F. R. Gambareli, M. D. Serra, L. J. Pereira, and M. B. D. Gavião, "Influence of measurement technique, test food, teeth and muscle force interactions in masticatory performance," Journal of Texture Studies, vol. 38, no. 1, pp. 2-20, 2007.

[16] S. R. Drago, M. Panouillé, A. Saint-Eve, E. Neyraud, G. Feron, and I. Souchon, "Relationships between saliva and food bolus properties from model dairy products," Food Hydrocolloids, vol. 25, no. 4, pp. 659-667, 2011.

[17] S. A. Rodrigues, A. K. Young, B. J. James, and M. P. Morgenstern, "Structural changes within a biscuit bolus during mastication," Journal of Texture Studies, vol. 45, no. 2, pp. 89-96, 2014.

[18] W. A. Alsanei, J. Chen, and R. Ding, "Food oral breaking and the determining role of tongue muscle strength," Food Research International, vol. 67, pp. 331-337, 2015.

[19] M. D. de Lavergne, C. Tournier, D. Bertrand, C. Salles, F. Van de Velde, and M. Stieger, "Dynamic texture perception, oral processing behaviour and bolus properties of emulsion-filled gels with and without contrasting mechanical properties," Food Hydrocolloids, vol. 52, pp. 648-660, 2016.

[20] M.-L. Jalabert-Malbos, A. Mishellany-Dutour, A. Woda, and M.-A. Peyron, "Particle size distribution in the food bolus after mastication of natural foods," Food Quality and Preference, vol. 18, no. 5, pp. 803-812, 2007.

[21] B. James, A. Young, B. Smith, E. Kim, A. Wilson, and M. P. Morgenstern, "Texture changes in bolus to the "point of swallow"-fracture toughness and back extrusion to test start and end points," Procedia Food Science, vol. 1, pp. 632-639, 2011.

[22] J. Chen and L. Lolivret, "The determining role of bolus rheology in triggering a swallowing," Food Hydrocolloids, vol. 25, no. 3, pp. 325-332, 2011.

[23] J. Gao, J. X. Wong, J. C.-S. Lim, J. Henry, and W. Zhou, "Influence of bread structure on human oral processing," Journal of Food Engineering, vol. 167, pp. 147-155, 2015.

[24] A. K. Young, J. N. Cheong, D. I. Hedderley, M. P. Morgenstern, and B. J. James, "Understanding the link between bolus properties and perceived texture," Journal of Texture Studies, vol. 44, no. 5, pp. 376-386, 2013. 

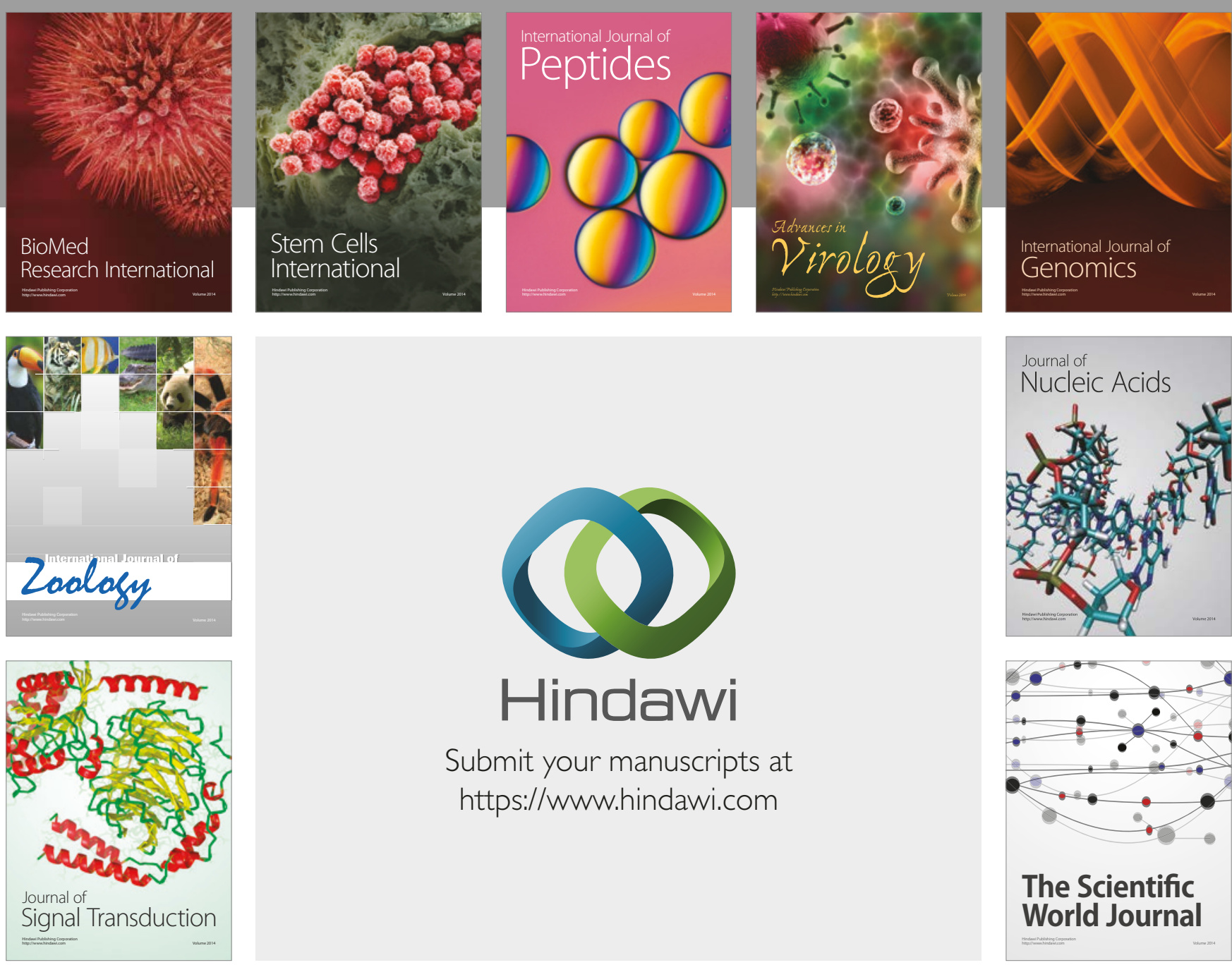

Submit your manuscripts at

https://www.hindawi.com
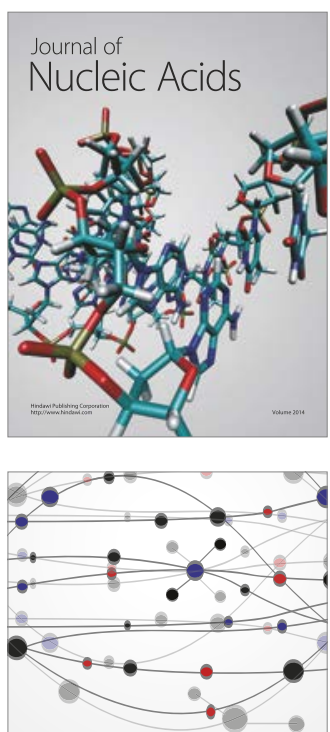

The Scientific World Journal

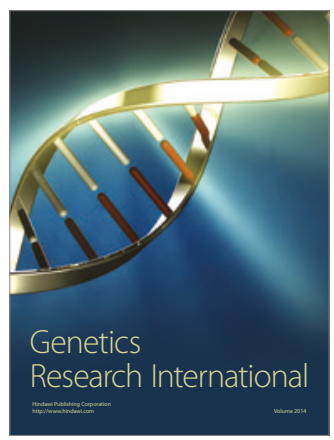

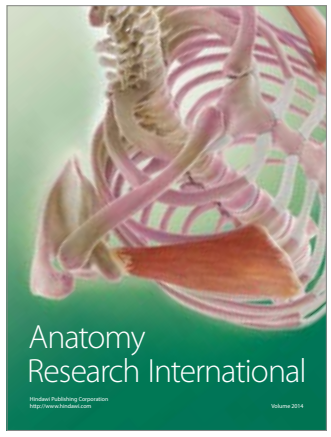

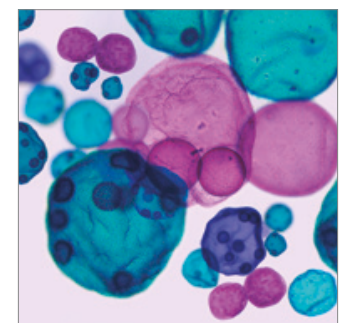

International Journal of Microbiology
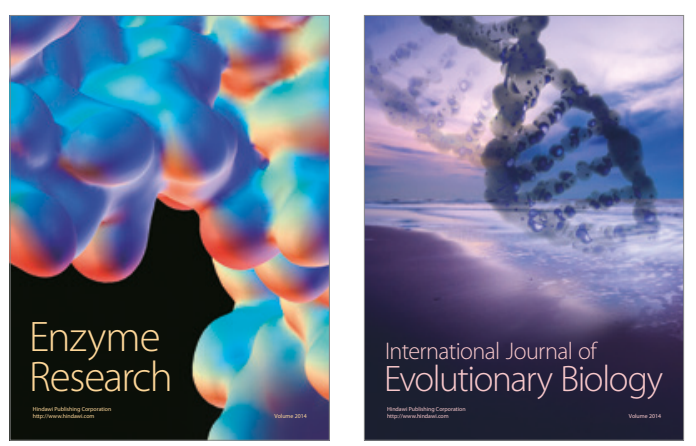
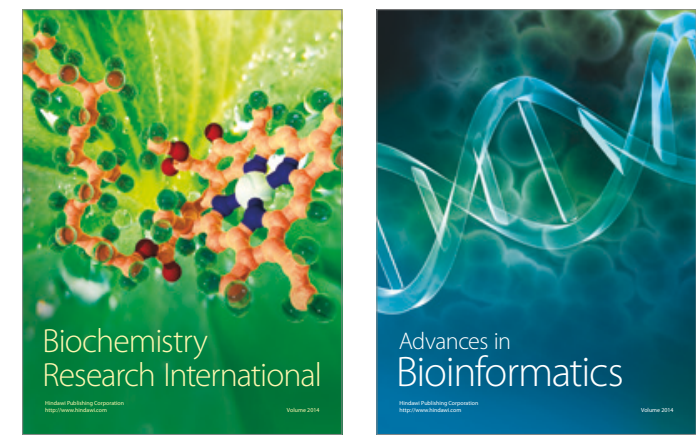

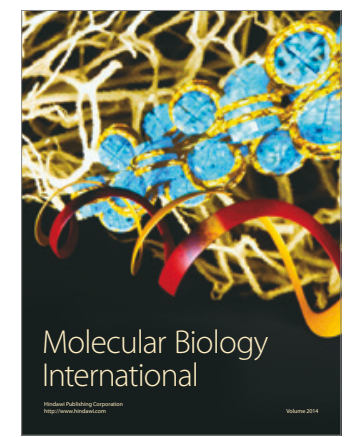

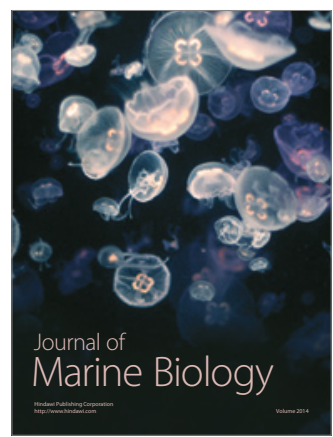

